\title{
The influence of visual motion on fast reaching movements to a stationary object
}

\author{
David Whitney ${ }^{\star}$, David A. Westwood ${ }^{\dagger}$, and Melvyn A. Goodale ${ }^{*}$ \\ ${ }^{*} \mathrm{CIHR}$ Group on Action and Perception, The Department of Psychology, The University of \\ Western Ontario, London, Ontario N6A 5C2, Canada \\ †School of Health and Human Performance, Dalhousie University, Halifax, Nova Scotia B3H 3J5, \\ Canada
}

\begin{abstract}
One of the most important functions of vision is to direct actions to objects ${ }^{1}$. However, every time that vision is used to guide an action, retinal motion signals are produced by the movement of the eye and head as the person looks at the object or by the motion of other objects in the scene. To reach for the object accurately, the visuomotor system must separate information about the position of the stationary target from background retinal motion signals-a long-standing problem that is poorly understood ${ }^{2-7}$. Here we show that the visuomotor system does not distinguish between these two information sources: when observers made fast reaching movements to a briefly presented stationary target, their hand shifted in a direction consistent with the motion of a distant and unrelated stimulus, a result contrary to most other findings ${ }^{8,9}$. This can be seen early in the hand's trajectory $(\sim 120 \mathrm{~ms})$ and occurs continuously from programming of the movement through to its execution. The visuomotor system might make use of the motion signals arising from eye and head movements to update the positions of targets rapidly and redirect the hand to compensate for body movements.
\end{abstract}

In the first experiment we investigated the role of distant motion signals in the updating of reaching movements to a stationary target. We briefly presented a stationary object while subjects fixated on a bull's-eye at the centre of a screen (see Fig. 1a and Methods). Subjects hit the position of the flashed object as quickly as possible with their index finger. A vertically drifting pattern was presented on the screen throughout the trial. Initially the pattern drifted in one direction, but, at an unpredictable moment, the direction of the drifting pattern was reversed.

Figure $1 \mathrm{~b}$ shows that the endpoints of the reaching movements were shifted either upwards or downwards in the direction of the nearby motion. Target flashes presented well before (for example $-940 \mathrm{~ms}$ ) or well after (for example $470 \mathrm{~ms}$ ) the motion reversal (at $0 \mathrm{~ms}$ ) produced systematic shifts in the hand's position. Because the target in these cases was presented sufficiently long before or after the motion reversal, the entire reaching movement -both programming and execution - was performed during unidirectional motion (average movement onset and movement duration were 224 and $262 \mathrm{~ms}$, respectively). For this reason it is unclear whether the motion influenced the programming phase or the on-line phase of the reaching movement, or both.

\section{(C) 2003 Nature Publishing Group}

Correspondence and requests for materials should be addressed to D.W. (dvw@uwo.ca).

Supplementary Information accompanies the paper on www.nature.com/nature.

Competing interests statement The authors declare that they have no competing financial interests. 
The target flash presented $235 \mathrm{~ms}$ before the moving pattern reversed direction addresses this question: because the average reaction time was $220 \mathrm{~ms}$, motion was in one direction during most of the programming phase and in the opposite direction during the movement. This condition produced a markedly reduced shift in the movement endpoint (grey oval in Fig. 1b). The only way in which the shift in the hand's endpoint could be reduced to this extent would be if the contributions of visual motion to programming and on-line control were approximately equal.

Fast reaching movements were subject to on-line control, which is consistent with previous studies in which the target's location is physically altered at the beginning of the reaching movement ${ }^{10-14}$. Because a continuously moving background was present in this first experiment, the data allow a close examination of how the representation of target location is updated over time and how this representation is used to adjust an ongoing response.

The left-hand side of Fig. 2a shows the average hand trajectory for one subject when there is downward visual motion near the target during the course of the reach (this sample trajectory is taken, in part, from the data point at $0 \mathrm{~ms}$ interstimulus asynchrony (ISA) for subject E.L.V. in Fig. 1b; initially the visual motion nearest the target was upwards, but when the target was presented the motion reversed direction, so that the reach was executed during downward motion only). When the trajectory for upward motion (right-hand side of Fig. 2a) is subtracted from this, giving the difference in hand position over time (Fig. 2b), it is clear that the hand continuously shifted in the direction of the nearby motion (increasingly negative values indicate a stronger influence of visual motion on hand position; see Supplementary Information for additional data).

One of the purposes of using a drifting pattern that reversed direction was to reveal the time course of the influence of motion on hand position. Figure 3a shows the average hand trajectories during upward and downward motion in response to the target presented $235 \mathrm{~ms}$ before the motion reversed direction (results are for the same subject as in Fig. 2). Figure $3 b$ shows the difference between these curves, which reveals the influence of the motion reversal on the hand's trajectory. In this case, visual motion is in one direction during most of the programming phase and in the opposite direction during the execution of the reaching movement. Interestingly, the trajectory of the hand follows the same pattern: the hand deviates in the direction of the initial motion and subsequently shifts in the opposite direction, mimicking the motion reversal.

Because there is a significant visuomotor delay, some time must pass before visual information can influence the trajectory of the hand. This delay can be estimated from the data in Fig. 3b. Immediately after movement onset $(236 \mathrm{~ms})$, the hand begins to deviate in the direction of the initial visual motion. The hand eventually starts to shift in the opposite direction (arrow in Fig. 3b), reflecting the influence of the motion reversal on the hand. The delay between the actual motion reversal (dashed vertical line at $235 \mathrm{~ms}$ ) and the moment that the data curve reaches a plateau ( $350 \mathrm{~ms}$ ) gives a minimum visuomotor delay of $\sim 114$ ms (see Supplementary Information for an alternative method of calculating the delay). The upper limit on the visuomotor delay is $\sim 201 \mathrm{~ms}$, based on the moment $(437 \mathrm{~ms})$ at which the average deviation of the hand's position differs significantly from that estimated from the minimum visuomotor delay $\left(t_{(73)}=2.05, P<0.05\right)$; this is consistent with the fact that the hand's relative position becomes negative at $437 \mathrm{~ms}$. (Estimates of visuomotor delay for additional subjects are provided in Supplementary Information). The range of this estimated visuomotor delay ( 114-201 ms) is similar to that for actual changes in target location $1,10,12,14,15$, indicating that the influence of visual motion on the updating of fast reaching movements occurs on the same time scale as actual changes in target position; that is, motion-generated position reassignment might be equivalent to a shift in the real position 
of the target ${ }^{15}$. This is surprising, because it indicates that information unrelated to the target (extraneous visual motion) might be processed as fast as information specific to the target, such as actual target location.

The present results show that visual motion information can cause shifts in fast reaching movements to the location of a briefly presented, unrelated stationary object. Previous studies have found that goal-directed reaching can, in some circumstances, be influenced by perceptual illusions, indicating that the awareness of a stimulus might determine the behavior ${ }^{15-22}$. Figure $3 b$ shows that this did not occur in the present experiment. The trajectory of the hand was modified continuously as the direction of visual motion changed. In this particular case, the hand first moved in the direction of the initial motion (for example upwards, after upward visual motion; the first significant deviation of the hand upwards occurred $\sim 35 \mathrm{~ms}$ after movement onset). However, the target flash is never perceived to be shifted upwards in this situation; the flash always appears either shifted downwards or not shifted at all ${ }^{7}$. The hand initially moved upwards, which is in a direction opposite to that of the perception. If reaching movements depended on awareness of the target's location, the hand should never have been shifted upwards; clearly visual motion influences the representation of target position for fast reaching movements without requiring explicit awareness of the target's position.

One possibility is that the visual motion influenced the perceived speed or position of the hand. To confirm that visibility of the hand is not necessary, we repeated the experiment while visibility of the hand was occluded. The results (see Supplementary Information) were similar to those of the first experiment (Fig 1b), indicating that the influence of motion on reaching is not due to the visual representation of hand speed or location.

The influence of visual motion on fast reaching movements is greatly reduced when there are significant cues to the target object's position. For example, when the duration of the target is increased sufficiently, the endpoints of the movements are accurate (Fig. 4a). This is an interesting situation, because when the visual information about the location of the target is first used to guide the reaching movement, the visuomotor system has no knowledge of the duration of the target; in other words, at the beginning of the programming phase a brief target flash is identical to a long one, as far as the motor system is concerned. Therefore, for long-duration targets we might expect the trajectory of the hand to deviate in the direction of the visual motion early in the movement, but then to correct itself as the duration of the target increases. This is precisely what we observed (Fig. 4b). During the initial phase of the reach, the surrounding motion signals influenced the position of the hand. However, when there was continued retinal information about target location, the representation of position was recalibrated and the hand's trajectory was updated on-line. The implication is that for any abruptly appearing object, even one that remains visible, there is an influence of visual motion on the hand's early trajectory. The influence of motion on fast reaching movements (revealed in the first experiment) is therefore not restricted to flashed targets that disappear long before the onset of the hand's movement.

Fast reaching movements ultimately depend on a comparison of target and hand position in a common coordinate system ${ }^{10,23,24}$, a comparison that is likely to be computed only on demand ${ }^{25}$. However, information about target location must initially be represented in retinotopic coordinates. If this early representation of space were influenced by motion, we would naturally expect subsequent processing that hinges on this information to manifest a similar distortion. Indeed, motion information is known to influence position coding early in visual processing, even at the level of the retina ${ }^{26}$ (and probably the cortex $2,4,5,7,27-29$ ). It is therefore conceivable that retinal motion signals, even those unrelated to the target, such as the vertically drifting grating in our experiments or the motion across the retina during eye 
and head movements, might cause continuous updating of target positions for reaching movements. Because the most common source of retinal motion is eye and head movements, an intriguing possibility is that the influence of visual motion on the representation of target position is an adaptive mechanism that allows the hand's trajectory to compensate rapidly for movements of the eye and head with respect to the world.

If visual motion is used to help compensate for ego-motion when executing actions, then we might expect the removal of motion cues to cause increased errors in goal-directed reaching movements. In an experiment, subjects reached to targets while they were passively rotated in a chair (that is, subjects did not have control over their rotation). This form of ego-motion was employed because visual motion might be especially useful for the compensation of passive (unintended) movements of the body. There were three conditions in this experiment. In the first, subjects reached to the remembered locations of targets (lightemitting diodes (LEDs)) while in complete darkness (no visual cues were available). Because this removes not only retinal motion information but also static position cues, a second condition presented intermittent background illumination at $\sim 4 \mathrm{~Hz}$ (the background consisted of 25 LEDs that were strobed; see Methods). This effectively eliminated motion information but left static position cues intact. In the third condition-the only condition in which motion information was available - the 25 background LEDs were continuously visible. When no background was visible, endpoints of the reaching movements were biased in the direction of the subject's rotation (subjects overshot the target, underestimating the magnitude of their rotation, which is consistent with previous studies ${ }^{30}$ ). There was a significant improvement (decrease) in this bias when retinal motion was available, compared with both the intermittent illumination condition $\left(t_{(136)}=4.4, P<0.001\right)$ and the condition in which no lights were visible $\left(t_{(132)}=6.4, P<0.001\right.$; see Supplementary Information). In other words, the background visual motion caused a deviation in the reaching movement, and this helped to counteract a bias towards underestimating the magnitude of ego-motion.

Although retinal motion information might be useful for controlling action in cases of whole-body rotation, could it be useful in cases where just the eye moves? Because the relative positions of the hand and target do not change during an eye movement, it seems that any influence of motion on reaching movements would be counterproductive. To test this, we conducted an experiment similar to the previous one, except that subjects moved their eyes (smooth pursuit) rather than rotating their entire bodies (see Supplementary Information). Results showed that pointing movements were more accurate when there was a stationary background (providing retinal motion as the subject moved his or her eyes) than when the background was intermittently visible or not visible. Retinal motion, produced by either eye or body movements, therefore reduces a systematic bias in reaching movements.

Reaching quickly to targets seems effortless despite the frequency and even the unintentionality of ego-motion. The experiments here suggest that part of the reason for this precision and speed might be the use of visual motion signals as a means of rapidly gauging and compensating for errors that occur when the eye or body moves.

\section{Methods}

Stimuli were presented on a CRTmonitor (NEC, Itasca, Illinois, USA) with a refresh rate of $85 \mathrm{~Hz}$, controlled by a Macintosh G4 computer (Apple Computer, Cupertino, California, USA). The stimulus consisted of a pair of horizontally striped patterns (gratings) that drifted vertically in opposite directions (Fig. 1a). Each grating subtended $5.76^{\circ} \times 26.76^{\circ}$, and each was centred at $\pm 5.76^{\circ}$ eccentricity. The gratings had a sinusoidal luminance modulation of 0.17 cycles per degree at $85 \%$ contrast on a dark background $\left(0.03 \mathrm{~cd} \mathrm{~m}^{-2}\right)$ and translated at $2.7 \mathrm{~Hz}$. Subjects were seated $48 \mathrm{~cm}$ from the monitor in a darkened room with a chin rest to 
stabilize the head. Two of the authors and two naive subjects participated in the first experiment (a total of six additional subjects participated in the subsequent experiments). Subjects were right-handed and had normal or corrected-to-normal visual acuity. Reaching movements were recorded, by using a single infrared emitter on the tip of the index finger, with an Optotrak 3020 (Northern Digital, Inc., Waterloo, Ontario, Canada) at $250 \mathrm{~Hz}$, under the control of the same computer that generated the stimuli.

A fixation bull's-eye was provided at the centre of the screen. In each trial there was a random vertical offset in the position of the bull's-eye within a range of $1.16^{\circ}$. Subjects were asked to fixate on the bull's-eye at all times. In each experimental trial, the two gratings (Fig. 1a) were initially stationary for $\sim 500 \mathrm{~ms}$ and then began to translate in opposite directions. The initial direction of the gratings' motion was random in each trial. After a randomly determined interval between 1,050 and 1,750 ms the gratings both abruptly reversed direction and translated for an equivalent period. At varying periods of time before or after the gratings reversed direction (ISA, from $940 \mathrm{~ms}$ before to $470 \mathrm{~ms}$ after the reversal), a target bar $\left(84.2 \mathrm{~cd} \mathrm{~m}^{-2}\right.$ ) was presented (as in Fig. 1a) for $\sim 24 \mathrm{~ms}$. The target subtended $2.89^{\circ} \times 0.29^{\circ}$ and could be located in one of three randomly determined positions, either vertically centred or $1.16^{\circ}$ above or below centre. The Optotrak began recording the position of the hand immediately after the target was presented. Subjects pointed, with their right index finger, as quickly as possible to the position of the target, hitting the CRT with their finger. The distance between the hand's resting position and the target on the monitor was $\sim 31-42 \mathrm{~cm}$. The onset of the reaching movement was calculated by using a threshold hand velocity $\left(50 \mathrm{~mm} \mathrm{~s}^{-1}\right.$ for $\left.20 \mathrm{~ms}\right)$. The end of the reaching movement was calculated as the moment that the hand's deceleration peaked (the subject hit the immobile CRT at a high velocity, and the resulting instantaneous deceleration of the hand marked the moment that the finger hit the screen). In the first experiment there were 90 trials for each of the seven possible ISAs. To measure average hand trajectories (in Figs 2-4) a minimum of 300 additional trials were collected in separate experimental sessions for each of the conditions of interest (the additional trials explain why the endpoints shown in Fig. $1 \mathrm{~b}$ are not identical to the average endpoints shown in Figs 2-4). The average trajectory was calculated as the normalized position of the hand, every $4 \mathrm{~ms}$ for each of the (three) target positions, as a function of motion direction.

In the last experiment, subjects were seated in a vestibular chair that rotated sinusoidally (through $\sim 90^{\circ}$ ) at a peak frequency of $\sim 0.2 \mathrm{~Hz}$. A chin rest was provided to immobilize the head. The head was located $20 \mathrm{~cm}$ from the axis of rotation. Subjects fixated on a red LED $(16 \mathrm{~cm}$ distance) that was attached to the head (so the eye was fixed with respect to the body). Vision was monocular and the room was completely dark; subjects could not see their hand under any of the conditions. While subjects were stationary, a 100-ms target LED ( $\sim 40 \mathrm{~cm}$ distance) was presented in one of five randomly determined locations. Subjects began to rotate $\sim 1 \mathrm{~s}$ later, clockwise and anticlockwise on alternate trials. At a randomly determined time (1.4-2.8 s after the start of rotation), subjects were instructed to point as quickly as possible to the remembered location of the target. Endpoints of the reaching movements were determined as described above. In one condition there were 25 additional LEDs in the background that were continuously visible throughout the experiment (see Supplementary Information). In a second condition, the background LEDs were strobed at $\sim 4 \mathrm{~Hz}$ (visible for $\sim 30 \mathrm{~ms}$, off for $\sim 200 \mathrm{~ms}$ ). In a third condition, there was no visible background. There were 100 trials in each of the three conditions. The mean endpoint position of the hand was calculated for each of the five target positions and two directions of rotation (reach error $=$ mean endpoint minus the actual location of the target). 


\section{Supplementary Material}

Refer to Web version on PubMed Central for supplementary material.

\section{Acknowledgments}

We thank J. Ladich, D. Pulham, C. Thomas, L. Van Cleeff, E. Veinsreideris and H. Yang. This work was supported by grants from US National Institutes of Health/National Eye Institute to D.W., and from Canadian Institutes of Health Research and the Canada Research Chairs Program to M.A.G.

\section{References}

1. Jeannerod, M. The Neural and Behavioural Organization of Goal-Directed Movements. Clarendon Press; Oxford: 1988.

2. De Valois RL, De Valois KK. Vernier acuity with stationary moving Gabors. Vision Res. 1991; 31:1619-1626. [PubMed: 1949630]

3. Kowler, E. Eye Movements and their Role in Visual and Cognitive Processes. Elsevier; Amsterdam: 1990.

4. Nishida S, Johnston A. Influence of motion signals on the perceived position of spatial pattern. Nature. 1999; 397:610-612. [PubMed: 10050853]

5. Ramachandran VS, Anstis SM. Illusory displacement of equiluminous kinetic edges. Perception. 1990; 19:611-616. [PubMed: 2102995]

6. Smeets JBJ, Brenner E. Perception and action are based on the same visual information: distinction between position and velocity. J Exp Psychol Hum Percept Perform. 1995; 21:19-31. [PubMed: 7707030]

7. Whitney D, Cavanagh P. Motion distorts visual space: shifting the perceived position of remote stationary objects. Nature Neurosci. 2000; 3:954-959.

8. Bridgeman B, Lewis S, Heit G, Nagle M. Relation between cognitive and motor-oriented systems of visual position perception. J Exp Psychol Hum Percept Perform. 1979; 5:692-700. [PubMed: 528967]

9. Wong E, Mack A. Saccadic programming and perceived location. Acta Psychol (Amst). 1981; 48:123-131. [PubMed: 7304220]

10. Desmurget M, Grafton S. Forward modeling allows feedback control for fast reaching movements. Trends Cogn Sci. 2000; 4:423-431. [PubMed: 11058820]

11. Goodale MA, Pelisson D, Prablanc C. Large adjustments in visually guided reaching do not depend on vision of the hand or perception of target displacement. Nature. 1986; 320:748-750. [PubMed: 3703000]

12. Paulignan Y, MacKenzie C, Marteniuk R, Jeannerod M. The coupling of arm and finger movements during prehension. Exp Brain Res. 1990; 79:431-435. [PubMed: 2323388]

13. Pelisson D, Prablanc C, Goodale MA, Jeannerod M. Visual control of reaching movements without vision of the limb. II Evidence of fast unconscious processes correcting the trajectory of the hand to the final position of a double-step stimulus. Exp Brain Res. 1986; 62:303-311. [PubMed: 3709715]

14. Prablanc C, Martin O. Automatic control during hand reaching at undetected two-dimensional target displacements. J Neurophysiol. 1992; 67:455-469. [PubMed: 1569469]

15. Brenner E, Smeets JBJ. Fast responses of the human hand to changes in target position. J Mot Behav. 1997; 29:297-310. [PubMed: 12453772]

16. Abrams RA, Landgraf JZ. Differential use of distance and location information for spatial localization. Percept Psychophys. 1990; 47:349-359. [PubMed: 2345688]

17. Bacon JH, Gordon A, Schulman PH. The effect of two types of induced-motion displays on perceived location of the induced target. Percept Psychophys. 1982; 32:353-359. [PubMed: 7155781]

18. Bridgeman B, Peery S, Anand S. Interaction of cognitive and sensorimotor maps of visual space. Percept Psychophys. 1997; 59:456-469. [PubMed: 9136275] 
19. Lepecq JC, Jouen F, Dubon D. The effect of linear vection on manual aiming at memorized directions of stationary targets. Perception. 1993; 22:49-60. [PubMed: 8474834]

20. Masson G, Proteau L, Mestre DR. Effects of stationary and moving textured backgrounds on the visuo-oculo-manual tracking in humans. Vision Res. 1995; 35:837-852. [PubMed: 7740774]

21. Sheth BR, Shimojo S. In space, the past can be recast but not the present. Perception. 2000; 29:1279-1290. [PubMed: 11219985]

22. Yamagishi N, Anderson SJ, Ashida H. Evidence for dissociation between the perceptual and visuomotor systems in humans. Proc R Soc Lond B. 2001; 268:973-977.

23. Andersen RA, Snyder LH, Bradley DC, Xing J. Multimodal representation of space in the posterior parietal cortex and its use in planning movements. Annu Rev Neurosci. 1997; 20:303-330. [PubMed: 9056716]

24. Buneo CA, Jarvis MR, Batista AP, Andersen RA. Direct visuomotor transformations for reaching. Nature. 2002; 416:632-636. [PubMed: 11948351]

25. Henriques DY, Klier EM, Smith MA, Lowy D, Crawford JD. Gaze-centered remapping of remembered visual space in an open-loop pointing task. J Neurosci. 1998; 18:1583-1594. [PubMed: 9454863]

26. Berry MJ 2nd, Brivanlou IH, Jordan TA, Meister M. Anticipation of moving stimuli by the retina. Nature. 1999; 398:334-338. [PubMed: 10192333]

27. Hayes A. Apparent position governs contour-element binding by the visual system. Proc R Soc Lond B. 2000; 267:1341-1345.

28. Snowden RJ. Shifts in perceived position following adaptation to visual motion. Curr Biol. 1998; 8:1343-1345. [PubMed: 9843685]

29. Whitaker D, McGraw PV, Pearson S. Non-veridical size perception of expanding and contracting objects. Vision Res. 1999; 39:2999-3009. [PubMed: 10664799]

30. Blouin J, Gauthier GM, van Donkelaar P, Vercher JL. Encoding the position of a flashed visual target after passive body rotations. NeuroReport. 1995; 6:1165-1168. [PubMed: 7662899] 
a
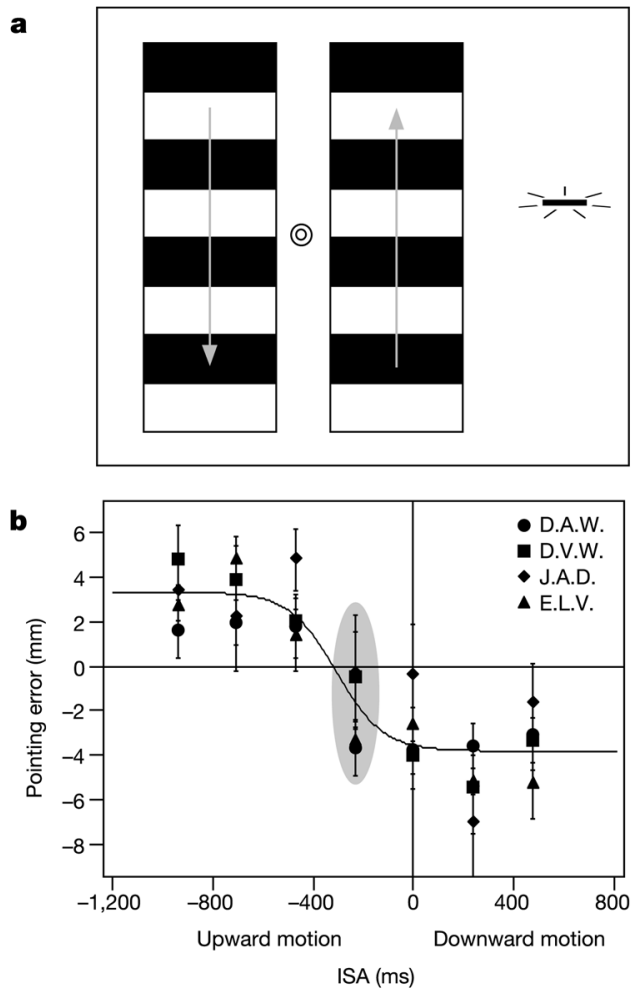

Figure 1.

Stimulus and results for the first experiment. a, Two patterns were presented that drifted vertically in opposite directions. The two patterns reversed direction after a randomly determined interval. Before or after this reversal, a stationary target was briefly presented near the pattern on the right. The target was stationary, rather than moving, because this allows a measurement of position without the confounding effects of target speed. $\mathbf{b}$, Results of the first experiment for four subjects. The abscissa shows the ISA between the flashed target and the motion reversal. (Negative ISA indicates that the target was presented before the motion reversal.) The motion reversal is depicted by the vertical line at 0 ISA. Data have been merged so that the motion nearest the target was upwards, then downwards, as indicated along the abscissa. The ordinate represents the magnitude of the hand's endpoint deviation, which was calculated as the average endpoint of the hand for initially upward motion trials minus initially downward motion trials (see Methods). Each data point shows the time at which the target was physically presented and the hand's endpoint deviation (although the data points show the time at which the targets were presented, reaching movements were completed $\sim 500 \mathrm{~ms}$ later). The influence of motion on the endpoint position of the hand varied systematically over time for each subject; the least significant effect was for subject J.A.D. $\left(F_{(6,77)}=3.14, P=0.008\right)$. The solid line is a sigmoid fit to the average of the four subjects' data. Results are means \pm s.e.m. 

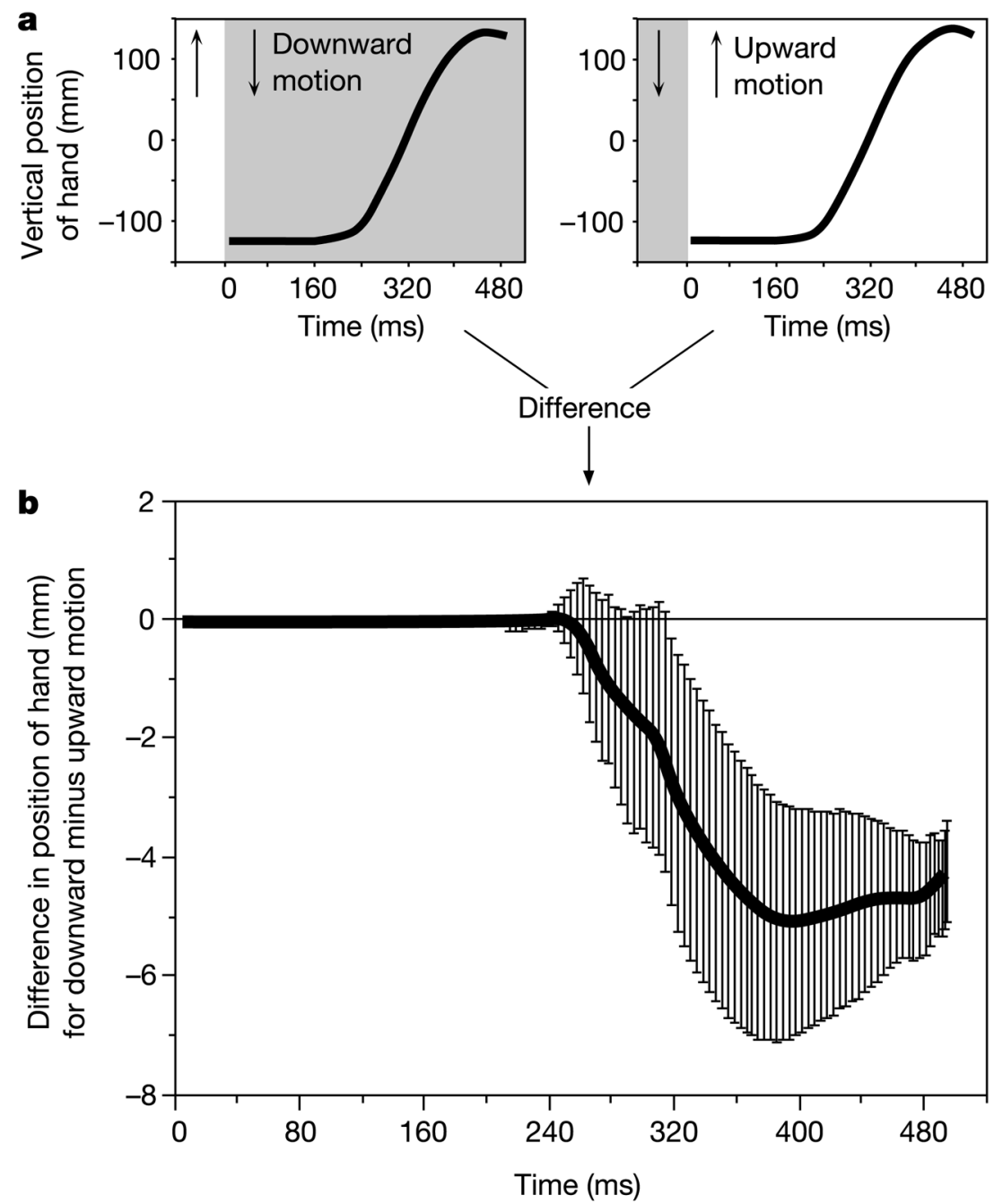

Figure 2.

Average hand trajectories from experiment 1 for subject E.L.V., where the target flash occurred coincidently with the motion reversal. a, The two trajectories show both sequences of visual motion (upwards followed by downwards, and vice versa). The grey region in each box represents the presentation of downward visual motion, and the white region indicates upward motion. The abscissa in each graph shows time; the target was presented at $0 \mathrm{~ms}$. The ordinate shows the hand's vertical position (the visual motion on the screen was drifting vertically, so we were interested in the vertical position of the hand). b. The hand's trajectory during upward motion in $\mathbf{a}$ is subtracted from that for downward motion to give the difference in hand position over time. The ordinate shows the shift in the hand's position: negative values indicate that the hand deviated in the direction of visual motion. In this example condition, the target was presented synchronously with the motion reversal ( 0 $\mathrm{ms}$ ), so the entire reaching movement was performed during unidirectional visual motion. There is a significant deviation in the trajectory of the hand as a function of time $\left(F_{(73,584)}=\right.$ $3.2, P<0.001)$. Results are means \pm s.e.m. 

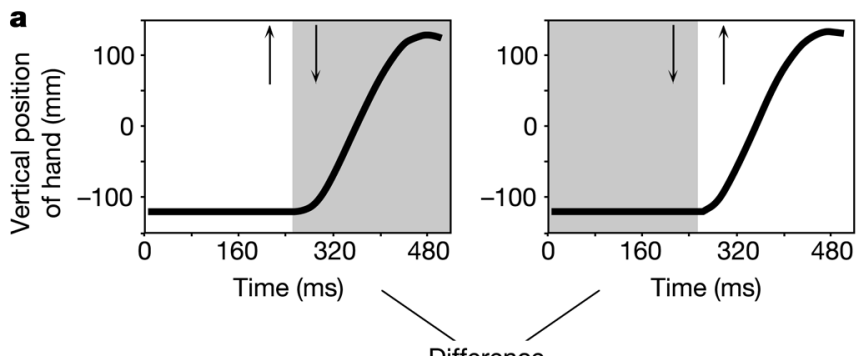

b

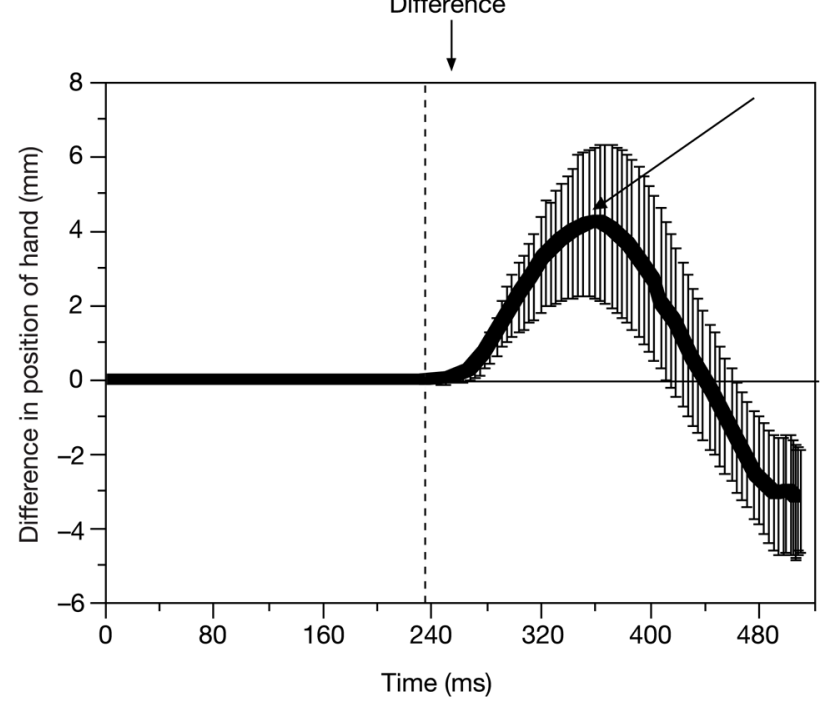

Figure 3.

Average hand trajectories from experiment 1 for subject ELV, where the target was presented $235 \mathrm{~ms}$ before the motion reversal. a, As in Fig. 2, the grey region in each box represents the presentation of downward motion, and the white region represents upward motion. The target was presented at $0 \mathrm{~ms}$. b. The trajectories of the hand for the two sequences of motion in a are subtracted (upward-downward minus downward-upward). The vertical dashed line represents the motion reversal. The ordinate shows the shift in the hand's position over time. The hand's position began to deviate in the initial direction of motion (increasingly positive values), but eventually the hand shifted in the opposite direction (arrow), which is consistent with the subsequent (reversed) direction of motion. There is a significant deviation in the trajectory of the hand as a function of time $\left(F_{(80,320)}=\right.$ $12.96, P<0.001)$. Results are means \pm s.e.m. 
a

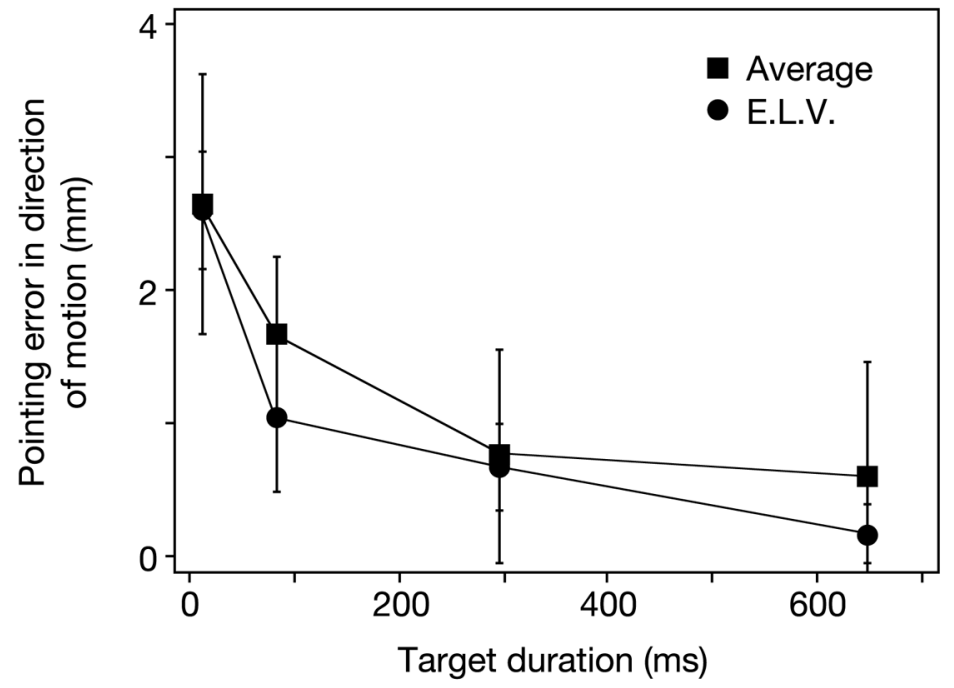

b

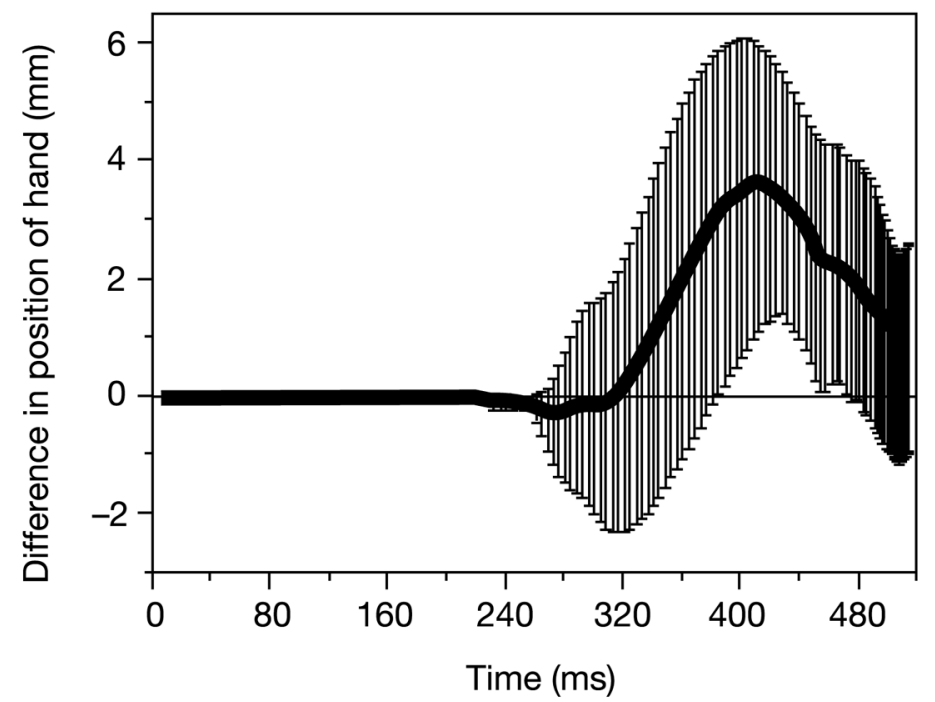

Figure 4.

Deviations in reaching movements as a function of target duration. a, The endpoints of reaching movements to briefly presented targets were shifted in the direction of visual motion. When the duration of the target exceeded $\sim 600 \mathrm{~ms}$, reaching movement endpoints did not exhibit the bias. Data for E.L.V. and the average for nine subjects are shown. b, The error in the hand's trajectory is plotted for the $640 \mathrm{~ms}$ target duration condition in a. See Supplementary Information for additional results. As in Figs $2 b$ and $3 b$, the difference in the hand's position for the two directions of motion is plotted. Positive values on the ordinate indicate that the hand deviated in the direction of visual motion. There is a significant overall effect of motion on the position of the hand $\left(t_{(299)}=4.97, P<0.001\right)$. Results are means \pm s.e.m. 